\title{
Displacement Whiplash PCR: Optimized Architecture and Experimental Validation
}

\author{
John A. Rose ${ }^{1,2,3, \star}$, Ken Komiya ${ }^{3,4}$, Satsuki Yaegashi ${ }^{3}$, and Masami Hagiya ${ }^{2,3}$ \\ ${ }^{1}$ Institute of Information Communication Technology, Ritsumeikan Asia Pacific University \\ jarose@apu.ac.jp \\ 2 Department of Computer Science and UPBSB, The University of Tokyo \\ hagiyalis.s.u-tokyo.ac.jp \\ ${ }^{3}$ Japan Science and Technology Agency-CREST \\ yaegashi@lyon.is.s.u-tokyo.ac.jp \\ ${ }^{4}$ Dept. of Computational Intelligence and Systems Science, Tokyo Institute of Technology \\ komiya@dis.titech.ac.jp
}

\begin{abstract}
Whiplash PCR-based methods of biomolecular computation (BMC), while highly-versatile in principle, are well-known to suffer from a simple but serious form of self-poisoning known as back-hybridization. In this work, an optimally re-engineered WPCR-based architecture, Displacement Whiplash PCR (DWPCR) is proposed and experimentally validated. DWPCR's new rule protect biostep, which is based on the primer-targeted strand-displacement of backhybridized hairpins, renders the most recently implemented rule-block of each strand unavailable, abolishing back-hybridization after each round of extension. In addition to attaining a near-ideal efficiency, DWPCR's ability to support isothermal operation at physiological temperatures eliminates the need for thermal cycling, and opens the door for potential biological applications. DWPCR should also be capable of supporting programmable exon shuffling, allowing XWPCR, a proposed method for programmable protein evolution, to more closely imitate natural evolving systems. DWPCR is expected to realize a highly-efficient, versatile platform for routine and efficient massively parallel BMC.
\end{abstract}

\section{Introduction}

In Whiplash PCR (WPCR), autonomous molecular computation is implemented by the recursive, self-directed polymerase extension of a DNA hairpin mixture [1]. When combined with a method for generating a combinatorial library of encoded strands, WPCR is theoretically capable of solving instances of a variety of NP-complete problems, including Inductive Inference [1] and Hamiltonian Path (HPP) [2]. WPCR has also been shown to be capable of supporting evolutionary computation, including: solution of HPP instances, via restriction-based crossover (Evolutionary WPCR (EWPCR)) [3]; co-evolution of poker strategies, via Parallel Overlap Assembly-based crossover [4]; and, programmable protein evolution, via EWPCR-based pseudo-module shuffling and RNA-protein fusion (XWPCR) [5].

\footnotetext{
* To whom correspondence should be addressed. 
(a) Whiplash PCR

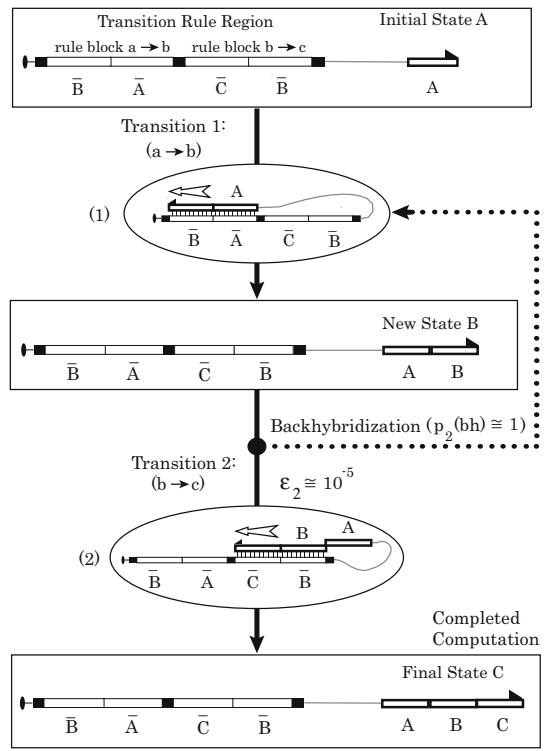

(b) Displacement Whiplash PCR

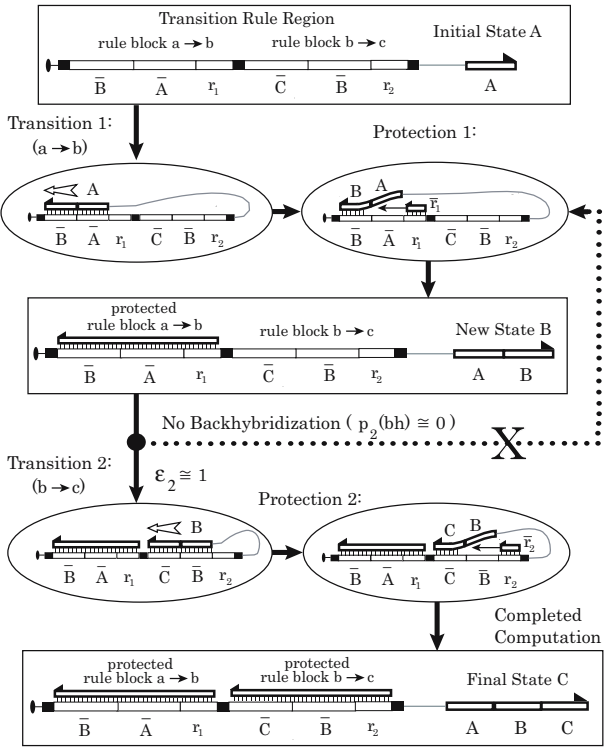

Fig. 1. (a) Whiplash PCR Top panel depicts a DNA strand encoding a WPCR program for executing the short computational path, $a \rightarrow b \rightarrow c$. Strand 3' and biotinylated 5' ends are indicated by black circles and arrowheads, respectively. Spacer and stop sequences are indicated by dark squares and light gray lines, respectively. In practice, efficient implementation is prevented by self-poisoning due to competitive hairpin formation, or back-hybridization (dotted line). (b) Displacement Whiplash PCR In DWPCR, encoded strands are re-engineered to support the addition of a back-hybridization eliminating rule protect step, which proceeds via primer annealing, extension, and strand displacement at target sequence, $r_{i}$, following transition, $i$.

Although the fundamental feasibility of using WPCR to implement multiple state transitions and test-scale instances has been demonstrated experimentally [2]67], a serious barrier confronting scaling for realistic application is a systematic tendency for WPCR strands to participate in a powerful form of self-inhibition called backhybridization [819]. Attempts to improve WPCR efficiency have involved both tuning of reaction conditions [6], and redesign of the basic WPCR architecture [8]. However, because the generation of highly-stable back-hybridized hairpins is favored energetically, elimination via either reaction condition optimization or strand-encoding strategies is impossible. Furthermore, the previous attempt to redesign the WPCR architecture to support targeted $\mathrm{PNA}_{2} / \mathrm{DNA}$ triplex formation (PWPCR [8]), thereby reducing the occupancy of back-hybridized structures was not fully optimal.

In this work, an optimally re-engineered WPCR-based architecture, Displacement WPCR (DWPCR) is proposed and experimentally validated. DWPCR provides a critical improvement for WPCR, by adding a new rule protect operation after each extension. This operation, which replaces thermal cycling, renders the most recently implemented rule-block of each computing strand unavailable via primer-targeted 
strand-displacement, virtually eliminating back-hybridization. In addition to achieving a near-ideal efficiency, DWPCR presents several other advantages, including: isothermal operation at physiological temperatures, opening the door for biological applications; and, the ability to support programmable exon shuffling, allowing XWPCR [5] to more closely imitate natural evolving systems. DWPCR is thus expected to realize a highly-efficient, versatile platform for routine and efficient massively parallel BMC.

Paper organization is as follows. Sec. 1.1 presents the original WPCR architecture, followed in Sec. 1.2 with a discussion of back-hybridization and proposal of the DWPCR architecture. Sec. 2 then presents a set of experiments designed to establish the fundamental feasibility of DWPCR, which rests on the efficiency of the key operation, rule protect. Sec. 3 3 presents experimental results, followed in Sec.4 4 with a discussion of experiment results, combinatorial DWPCR implementation, and potential applications.

\subsection{The Whiplash PCR Architecture}

The original WPCR architecture [1] is illustrated in Fig 1(a). As shown, state transitions are implemented via the recursive template-directed polymerase extension of a DNA hairpin mixture. For clarity, this process is described for a 3-state, 2 step implementation, $a \rightarrow b \rightarrow c$. Prior to computation, a single-stranded (ss) DNA molecule is encoded with three regions (top panel). The transition rule region contains a set of state transition rule-blocks, each of which encodes a transition from computational state $\mathrm{x}$ to state y. If $X$ and $\bar{X}$ respectively denote the DNA word for state $\mathrm{x}$, and its Watson-Crick reverse complement, then the rule-block for executing transition $x \rightarrow y$ is formed by catenated words, $5^{\prime}-\bar{Y} \bar{X}-3^{\prime}$. The completed transition rule region is formed by the concatenation of all rule-blocks, separated by short DNA stop sequences. In Fig. 1] the transition rule region contains rule-blocks for transitions $a \rightarrow b$ and $b \rightarrow c$. The 3'-most codeword (word A (top panel)) is the head, which encodes the initial state of the WPCR strand. A short DNA spacer sequence is placed between these two regions.

Each WPCR state transition is implemented via a three-step process of hybridization, polymerase extension, and denaturation, shown in Fig. 11a). Each transition is initiated via hybridization of the 3 ' head with a complementary DNA word in the transition rule region. The transition encoded by the hybridized rule-block is then executed via polymerase extension of the head (horizontal arrow in each transition), which appends the DNA word for the transition target state to the strand's 3' end. Polymerase extension is terminated automatically when the DNA polymerase encounters the transition block's 5' stop sequence, usually implemented using a short stop sequence (AAA), combined with the absence of free dTTP in the buffer. Following thermal denaturation of the newly-extended hairpin, the strand is ready for the next round of hybridization and extension (process $b \rightarrow c$ ). Upon completion of all encoded transitions, a record of the computed path is encoded at the strand's 3' end, as an ordered string of DNA words $\left(5^{\prime}-A B C-3^{\prime}\right.$ in Fig. 1 (bottom). As each strand is encoded to execute a distinct set of transition rules autonomously, massively parallel computation may be achieved by generating a combinatorial mixture of WPCR strands, and iteratively applying a thermal cycle appropriate for the parallel hybridization, extension, and denaturation of all strands. 


\subsection{Back-Hybridization and Displacement WPCR}

Unfortunately, WPCR state transition efficiency is compromised by a serious form of self-poisoning, known as back-hybridization. This effect is illustrated in Fig.11 a; dotted line) via formation of the extended hairpin structure (hairpin (1)), generated via transition, $a \rightarrow b$, which competes with formation of the extendable structure, (unextended hairpin (2)) required for transition $b \rightarrow c$. The effect of back-hybridization has been modeled theoretically [8], and shown to be sufficiently serious to render massivelyparallel WPCR infeasible. Experimental verification of basic model predictions for competitive hairpin formation has also been undertaken [9]. Back-hybridization is fundamentally less tractable than mis-hybridization (i.e., error duplex formation), in that the availability of back-hybridized structures in all rounds after the first is not an error. As a result, while mis-hybridization may be effectively minimized via strand-encoding strategies, difficulties due to back-hybridization will remain, regardless of the encoding method employed. For this reason, the fundamental re-engineering of the WPCR architecture is considered to be required.

The re-engineering of the WPCR architecture to support the primer-directed conversion of each implemented rule-block to double-stranded (ds) DNA, via targeted primerdirected strand displacement after each round of extension, is here proposed. The essential details of the resulting WPCR-based architecture, here referred to as Displacement WPCR (DWPCR), are illustrated in Fig. 22 DWPCR implements the same series of basic biotechnological operations applied in standard Whiplash PCR, adding only a protection step, which proceeds via primer annealing, extension, and strand displacement at target sequence, $r_{i}$, following each successful transition, $i$. Given use of an excess of DNA primer, and a DNA polymerase with high strand-displacement activity, such as the Klenow fragment of DNA polymerase I (see [10]), this protection operation is expected to result in the high efficiency conversion of the targeted rule-block to dsDNA, virtually eliminating the potential for back-hybridization in each successive round.

\section{Materials and Methods}

\subsection{Strand Design and Characterization}

The feasibility of high-efficiency DWPCR rests upon that of the rule protect biostep, which employs primer-directed opening of a DNA hairpin via strand displacement by DNA polymerase. Although the ability of a number of polymerases lacking 5' exonuclease ability (e.g. the Klenow fragment of DNA polymerase I) to displace an encountered strand during extension is well-known, the efficiency of using this process for opening a hairpin loop, representing a computational step should be explicitly established. Characterization of this process was accomplished via observation of the FRET system shown in Fig. 11 which consists of two DNA strands. Strand (a) is an 78 nucleotide (nt) template strand, which was encoded so that the 5' and 3'-most 15 bases (codewords 1 and 1', respectively) were Watson-Crick complementary, to facilitate formation of a 15 base-pair (pb) hairpin (panel (c)). To support observation of the binary folding state of this hairpin, the fluorophores FAM and tetramethylrhodamine (TAMRA) were covalently attached to the strand's 5' and 3' ends, respectively. Codewords 1-4 were 


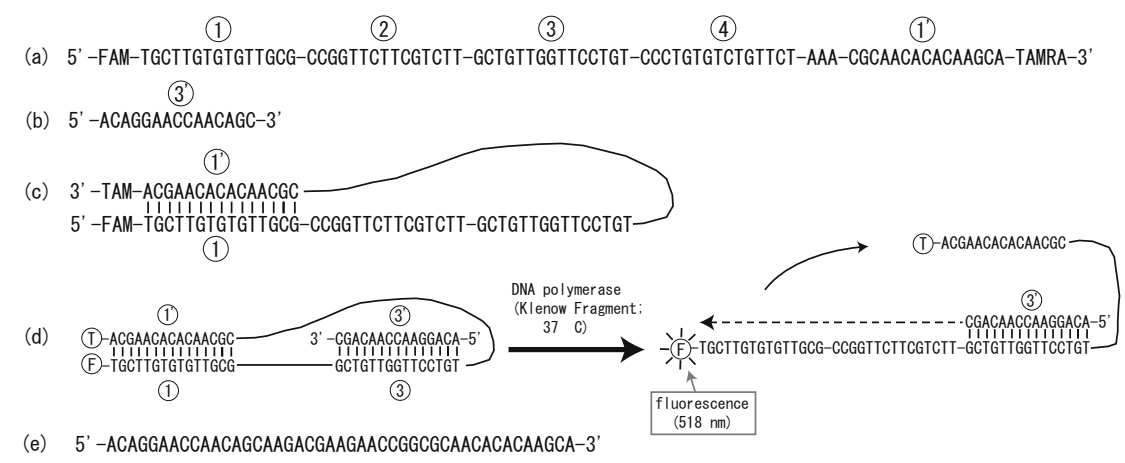

Fig. 2. Model Hairpin System for validating the Rule Protect operation (a) The hairpinforming template strand, composed of catenated DNA words 1, 2, 3, 4, and 1', shown separated with intervening dashes for clarity. This strand is 5' and 3'-substituted with FAM and TAMRA fluorescent groups, respectively; (b) primer strand, complementary to template codeword 3; (c) hairpin formed by normal closure of the template strand. Here, blunt-end duplex formation places the FAM/TAMRA pair in close contact, resulting in loss of FAM fluorescence at $518 \mathrm{~nm}$ by FRET or contact quenching; (d) the hairpin displacement process under study, directed by hybridization of primer strand at template codeword 3 , followed by primer extension. Successful primer extension, with full penetration into the template hairpin stem is accompanied by hairpin opening, with an observable increase in FAM fluorescence at $518 \mathrm{~nm}$; (e) the fully-extended primer.

derived from those employed in [7], designed to avoid mis-hybridization according to the strategy in [11]. Strand (b) is a $15 \mathrm{nt}$ primer strand (sequence 3') Watson-Crick reverse complementary to bases 46-60 (codeword 3 ) of the template strand. DNA strands $(\mathrm{a}, \mathrm{b})$ and strand $(\mathrm{c})$ were purchased from Nippon EGT and Sigma-Genosys, respectively (strand (c) was kindly gifted from M. Yamamura, Tokyo Institute of Technology).

\subsection{System Operation}

The essential idea of the hairpin test system is shown in Fig. 2 In the absence of primer and DNA polymerase, hairpin closure is assured by a reaction temperature beneath the characteristic $T_{m}$ value of template strand (a). Upon excitation of the FAM fluorophore at $494 \mathrm{~nm}$, the typical FAM fluorescence at $518 \mathrm{~nm}$ will be inhibited by FRET or contact quenching [12], due to the close proximity of TAMRA, provided by the blunt-end duplex design (d; left). Followed by the addition of the primer strand, and DNA polymerase (Klenow fragment of DNA polymerase I), at $37^{\circ} \mathrm{C}$ and under appropriate buffer conditions, successful primer extension (d; right) resulting in the hairpin displacement characteristic of DWPCR will be directly observable by the fluorescence of the excited fluorophore (FAM). On the other hand, failure of the hairpin displacement operation will be observable by the lack of a change in fluorescence, due to the maintenance of FRET and contact quenching. Panel (e) illustrates the fully-extended primer, which was also commercially synthesized separately, for comparison purposes.

A preliminary analysis was performed to ensure that the melting temperatures $\left(T_{m}^{\prime} s\right)$ of the template hairpin (c) and primer-template pair (d; right) were substantially above 
the planned experimental temperature of $37^{\circ} \mathrm{C}$, at reaction conditions of interest. The thermal stability of the template hairpin was characterized via a standard DNA melting curve obtained by observing the fluorescence of SYBR Green $\AA$ I (Molecular Probes) using an Real-time PCR machine OPTICON2 (Bio-rad), yielding a hairpin $T_{m}$ of $78.2^{\circ} \mathrm{C}$, at an ionic strength of $0.165 \mathrm{M}$ (data not shown). To avoid difficulties with multiple curve resolution, the thermal stability of the primer-target pair was investigated via simulation using the software package, NucleicPark [13] which employs the parameters in [14], with an enhanced statistical zipper model. Simulations yielded a primer-template $T_{m}$ of $52.3^{\circ} \mathrm{C}$, and a transition width of $\Delta T_{m}=6.8^{\circ} \mathrm{C}$ at an ionic strength of $0.165 \mathrm{M}$ and [template $]=$ [primer $]=0.1 \mu \mathrm{M}$. These results were taken as sufficient to indicate proper formation of the expected DNA helices, at reaction conditions of interest.

\subsection{Hairpin Displacement Experiments}

Hairpin-displacement via primer extension by DNA polymerase was performed and observed, using a fluorescence spectrophotometer F-2500 (Hitachi) with a compact lowtemperature thermostats RC6CP (Lauda), upon excitation at the characteristic FAM absorbance frequency $(494 \mathrm{~nm})$. A polyacrylamide gel electrophoresis (PAGE) experiment was also performed to visualize the variation in the size of the primer-mediated strand-displaced products. A set of wavelength fluorescence scans on polymerization was first performed to investigate the basic feasibility of primer-directed strand displacement and hairpin opening. A $0.1 \mu \mathrm{M}$ final concentration of the template hairpin was prepared in a $300 \mu \mathrm{l}$ reaction buffer consisting of $1 \mathrm{X}$ Klenow buffer $(50 \mathrm{mM} \mathrm{NaCl}$, $10 \mathrm{mM}$ Tris- $\mathrm{HCl}, 10 \mathrm{mM} \mathrm{MgCl}_{2}, 1 \mathrm{mM}$ DTT), $2 \mathrm{mM}$ dNTPs and $0.1 \mu \mathrm{M}$ strand displacement primer. This mixture was incubated at $37^{\circ} \mathrm{C}$, and subsequently, 5 units of DNA Polymerase I Klenow Fragment (New England BioLabs) was added, as appropriate. The fluorescence spectrophotometer excitation and emission slit widths were each set to $5 \mathrm{~nm}$. The fluorescence of the following samples were tested: blank (B; buffer only); a template-only sample ( $\mathrm{T}$; strand (a) and buffer (no primer)); a template-primer hybridized sample ( $\mathrm{T}+\mathrm{P}$; strands (a), (b), and buffer); and a template/primer/polymerase sample ( $\mathrm{T}+\mathrm{P}+\mathrm{K}$; strands (a), (b), Klenow Fragment, and buffer). Samples other than strand displaced template-primer samples were used as controls. The applied scan range was from $450 \mathrm{~nm}$ to $650 \mathrm{~nm}$, and the scan speed was set to $3000 \mathrm{~nm} / \mathrm{min}$. For the $\mathrm{T}+\mathrm{P}+\mathrm{K}$ run, the spectral scan measurement was repeated at various time points, beginning from $5 \mathrm{sec}$ after adding Klenow Fragment, to 10 min thereafter.

Secondly, a time-scale fluorescence scan on polymerization was performed to investigate the rapidity of the formation of extended product. The change in the fluorescence of the excited fluorophore (FAM) accompanying hairpin opening was measured at the emission wavelength of $518 \mathrm{~nm}$, in real time. The T+P mixture without Klenow Fragment was first monitored at $37{ }^{\circ} \mathrm{C}$ to measure the baseline of the fluorescence for the first $90 \mathrm{sec}$. The time scan was then continued for around 1 hour, after which 5 units of Klenow DNA polymerase was added. To investigate the impact of target saturation with primer strand, this experiment was repeated with a ten-fold saturation of primer strand (i.e., a total primer concentration of $1.0 \mu \mathrm{M}$ ).

A third polymerization experiment was performed to independently confirm the production of extended DNA product via PAGE, as follows. Polymerization was performed 
in $50 \mu \mathrm{l}+\mathrm{T}+\mathrm{K}$ mixture under incubation at $37{ }^{\circ} \mathrm{C}$, and then halted after various incubation time periods $(5,10,20,30,60$, and $600 \mathrm{sec})$, by adding the equal volume of Phenol:Chloroform:Isoamyl alcohol (25:24:1) (Nacalai Tesque) to the sample. Samples were then run on a $12 \%(\mathrm{w} / \mathrm{v})$ polyacrylamide gel.
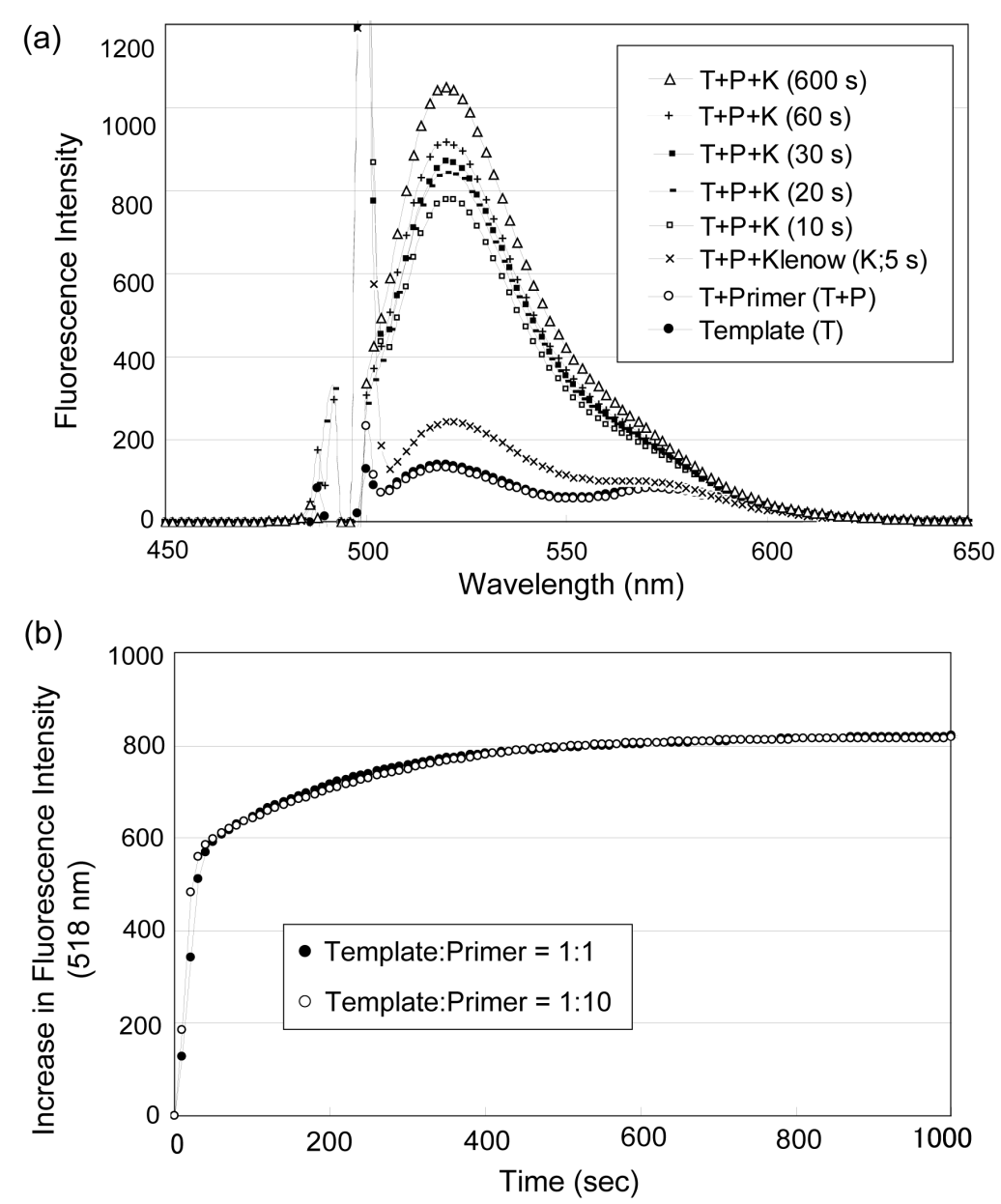

Fig. 3. Experimental Results (a,b) Fluorescence wavelength and time scans from the first and second polymerization experiments, respectively. See text for discussion.

\section{Results}

Fig. 3 (a) illustrates the results of the first polymerization experiment, and the resulting set of fluorescence wavelength scans. The fluorescence peak at $518 \mathrm{~nm}$, obtained in all curves is characteristic of FAM emission, stimulated via excitation at $494 \mathrm{~nm}$, with the accompanying spike at $494 \mathrm{~nm}$ indicative of scattering of the incident excitation beam. 
The smaller peak at $580 \mathrm{~nm}$ is due to TAMRA (via FRET transfer from FAM, and reemission). For runs without DNA polymerase (runs $\mathrm{T}$ and $\mathrm{T}+\mathrm{P}$ ), equally-low fluorescence values were obtained at $518 \mathrm{~nm}$, indicating FRET or contact quenching, resulting from the closure of hairpin (c) in Fig 2 . However, following addition of Klenow fragment, even minimal polymerization time is accompanied by a sizable increase in the fluorescence signal at $518 \mathrm{~nm}$ ( $5 \mathrm{sec}$; yellow curve). Additional polymerization time is accompanied by successively higher peaks, which rapidly reach saturation, by $600 \mathrm{sec}$. This result is consistent with the rapid abolition of FRET/TAMRA contact quenching, indicative of successful primer extension, strand displacement, and hairpin opening.

Fig. 3 (b) illustrates the results of the second polymerization experiment, in terms of the fluorescence increase of the excited fluorophore (FAM; $518 \mathrm{~nm}$ ) vs. time. As in the above experiment, the accompanying fluorescence time scan indicates that addition of Klenow fragment is accompanied by a rapid increase in fluorescence at 518 $\mathrm{nm}$, reaching saturation within 600 seconds, for both equimolar and a ten-fold excess of primer:template. This result is again consistent with the rapid abolition of FRET/ TAMRA contact quenching, indicative of successful primer extension, strand displacement, and hairpin opening, with essential completion within 600 seconds.

Fig. 4illustrates the PAGE gel resulting from the third polymerization experiment. The band with high mobility in the control run (lane 7; Template+Primer only; bottom arrow), which runs just under $80 \mathrm{bps}$, as judged via comparison with the $20 \mathrm{bp}$ ladder (lane 8) is indicative of unpolymerized DNA product (i.e., $\mathrm{T}+\mathrm{P}$ ). On the other hand, lanes 1 (immediate inactivation of Klenow primer) through 6 (600 sec of polymerization by Klenow primer) indicate the essentially immediate disappearance of the band with high mobility beneath $80 \mathrm{bps}$ (difficult to see after only $5 \mathrm{sec}$ of polymerization (lane 1)), and the rapid appearance of a band with lower mobility, running at $80 \mathrm{bps}$ (top arrow), which is consistent with the increasing accumulation of polymerized DNA product (lanes 1-6). To independently verify the identity of the band at 80 bps as the fully-extended product of polymerase extension, the mobility of the hybridized template and fully-extended primer was also investigated separately, and found to run at 80 bps (top arrow), as required (data not shown).

\section{Discussion}

The presented experimental results provide an unambiguous validation of the fundamental feasibility of targeted primer-directed hairpin displacement. Both the FRET and PAGE experiments indicate the rapid attainment of a high degree of completion of dsDNA product, corresponding to the hybridized tempate/extended-primer pair. In DWPCR, this basic operation, employed as the Rule Protect biostep, not only achieves hairpin displacement, but also abolishes back-hybridization by converting the most recently executed rule-block of all strands in an encoded mixture to double-stranded form, forcibly preventing hairpin formation within this region. As the template/extendedprimer duplex which forms the protected rule-block will clearly remain stable during the isothermal operation characteristic of DWPCR (thermal cycling has been eliminated) the experimental validity of DWPCR as a high-efficiency method for implementing massively parallel autonomous computation is considered to be firmly established, as well. 


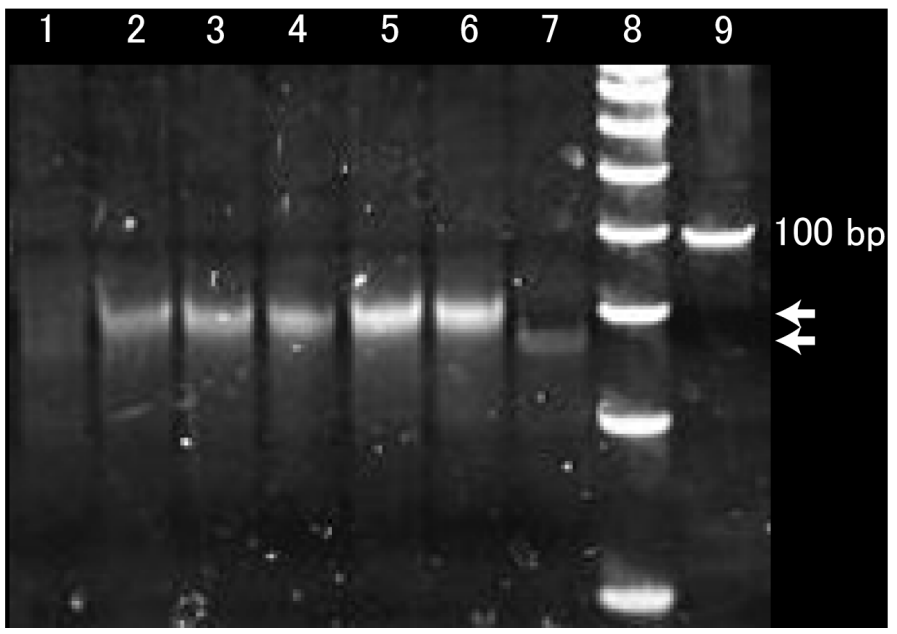

Fig. 4. Experimental Results PAGE gel from the third polymerization experiment. Lane 1-6: $\mathrm{T}+\mathrm{P}+\mathrm{K}$, with polymerization inactivated just after $(5 \mathrm{sec})$ and following addition of Klenow fragment at 10, 20, 30, 60, and $600 \mathrm{sec}$, respectively. Lane 7: Control ( $\mathrm{T}+\mathrm{P}$ only; no Klenow). Lane 8: 20 bp ladder. Lane 9: 100 bp DNA ladder. See text for discussion.

Given that the per-transition efficiencies estimated for WPCR and PWPCR are very low (roughly $10^{-5}$ and $10^{-2}$ successful transitions per polymerase-hairpin encounter), the per-transition efficiency approaching unity achieved by DWPCR for successfully protected rule-blocks represents a fundamental advance. Completion appears to be rapid and substantial. In contrast with WPCR, which exhibits low reaction efficiency, and requires a high temperature ofr operation $\left(e . g ., 80^{\circ} \mathrm{C}\right)$, DWPCR could proceed with an efficiency of nearly unity under physiological conditions $\left(37^{\circ} \mathrm{C}\right)$, and thus open the door for practical biological applications. Further investigation regarding a determination of the absolute concentration of fully-extended product is our concern. Issues related to a quantitative determination of efficiency are deferred to later work.

Implementation of the rule protect biostep to achieve high-efficiency DWPCR-based massive parallelism requires a method for preparing a combinatorial mixture of encoded DWPCR strands. Although in principle, various methods could be employed, the 5' to 3' ordering of transition rules provided by the combinatorial assembly method presented for PWPCR in [5] provides a number of clear advantages. First of all, this ordering ensures the round-by-round shortening of the hairpin loops required for successive state transitions on all strands. In this case, computational efficiency for each strand should increase as the computation progresses. Secondly, the accompanying segregation of successively-generated, protected rule-blocks towards each strand's 5' end avoids potential difficulties due to loop stiffness due to duplex islands within the loops used for hairpin computation. Finally, as noted in [5], this ordering supports implementation of a properly coordinated cross-over operation, to generate valid daughter strands in WPCR-based processes evolutionary computation (i.e.,, EWPCR and XWPCR. Accordingly, a 5' to 3' transition rule ordering is adopted by the DWPCR architecture. 
A striking secondary advantage of DWPCR over PWPCR regards its fundamentally superior ability to support WPCR-based evolutionary protein design. In particular, the polymerization of target sequences for PNA binding between state codeword pairs in the growing 3' tail compromises PWPCR's utility for evolutionary protein design by pseudo-module shuffling (XWPCR) [5]. As RNA-protein fusion-based expression of the $3^{\prime}$ tail as a single, unbroken gene is required by XWPCR, the unavoidable presence of glycine loops resulting from translation of transcribed target sequences, left over from the PWPCR process is strongly destabilizing to shuffled polypeptides (see [5] for a discussion). In contrast, target sequences for primer extension in DWPCR are within the rule-region rather than the growing 3' tail, so that destabilization due to the translation of targeting artifacts from the computational process will be completely absent, which itself constitutes a major advance. Furthermore, the architectural freedom gained by the absence of these transcribed glycine coils, which necessitates systematic placement at pseudo-module boundries in PWPCR-based XWPCR, will also allow DWPCR-based XWPCR to support exon shuffling (i.e., protein module rather than pseudo-module shuffling), enabling XWPCR to more closely imitate naturally-evolving systems. Thus, the ability to employ DWPCR, rather than PWPCR as the underlying state transition method represents a significant advance for XWPCR.

The fundamental primer-directed hairpin displacement operation should also be useful for non-WPCR DNA computing applications. For instance, this operation might be employed, rather than simple primer displacement, to direct state transitions in alternative hairpin-based architectures [15]. Beyond directing state transitions, this operation is also useful for parallel-processing of single-stranded DNA molecules during and after computation, such as in directing the conversion to dsDNA form following completed computation, required for gene expression in XWPCR [5]. The current fundamental operation is thus taken to have significance beyond simple WPCR-based applications.

\section{Acknowledgements}

Financial support generously provided by Grants-in-Aid for Scientific Research (Kiban B, 15300100 and 18300100), from the Japan Society for the Promotion of Science (JSPS) and by JST-CREST. The authors are also grateful to M. Yamamura of the Tokyo Institute of Technology for the kind gift of primer strand (c).

\section{References}

1. Hagiya, M., Arita, M., Kiga, D., Sakamoto, K., Yokoyama, S.: Towards parallel evaluation and learning of boolean $\mu$-formulas with molecules. In Rubin, H., Wood, D., eds.: DNA Based Computers III. (2000) 57-72

2. Sakamoto, K., Kiga, D., Komiya, K., Gouzu, H., Yokoyama, S., Ikeda, S., Sugiyama, H., Hagiya, M.: State transitions by molecules. Biosystems 52 (1999) 81-91

3. Rose, J.A., Hagiya, M., Deaton, R.J., , Suyama, A.: A DNA-based in vitro genetic program. J. Biol. Phys. 28 (2002) 493-498

4. Wood, D., Bi, H., Kimbrough, S., Wu, D.J., Chen, J.: DNA starts to learn poker. In: DNA Computing. $7^{\text {th }}$ Int'l Workshop on DNA-Based Computers. (2002) 22-32 
5. Rose, J.A., Takano, M., Hagiya, M., Suyama, A.: A DNA computing-based genetic program for in vitro protein evolution via constrained pseudomodule shuffling. Journal of Genetic Programming and Evolvable Machines 4 (2003)

6. Komiya, K., Sakamoto, K., Gouzu, H., Yokohama, S., Arita, M., Nishikawa, A., Hagiya, M.: Successive state transitions with I/O interface by molecules. In Condon, A., Rozenberg, G., eds.: DNA Computing. $6^{\text {th }}$ Int'1 Workshop on DNA-Based Computers. Volume 2054., Springer-Verlag, Berlin (2001) 17-26

7. Komiya, K., Sakamoto, K., Kameda, A., Yamamoto, M., Ohuchi, A., Kiga, D., Yokoyama, S., Hagiya, M.: DNA polymerase programmed with a hairpin DNA incorporates a multipleinstruction architecture into molecular computing. Biosystems 83 (2006) 18-25

8. Rose, J.A., Deaton, R.J., Hagiya, M., Suyama, A.: Equilibrium analysis of the efficiency of an autonomous molecular computer. Phys. Rev. E 65 (2002) 1-13 Article 021910.

9. Komiya, K., Yaegashi, S., Suyama, A., Hagiya, M., Rose, J.A.: Experimental validation of the statistical thermodynamic model for prediction of the behavior of autonomous molecular computers based on DNA hairpin formation. In: DNA Computing. $12^{\text {th }}$ Int'l Workshop on DNA-Based Computers. (2006) in press

10. New England Biolabs: Klenow Fragment ( $3 L \rightarrow 5 L$ exo-). (2004) Technical Bulletin M0212.

11. Arita, M., Nishikawa, A., Hagiya, M., Komiya, K., Sakamoto, K., Gouzu, H., Yokoyama, S.: Improving sequence design for DNA computing. In: Proc. $5^{t} h$ Genetic and Evolutionary Computation Conference (GECCO99), Las Vegas (2000) 875-82

12. Marras, S., Kramer, F., Tyagi, S.: Efficiencies of FRET and and contact-mediated quenching in oligonucleotide probes. Nucl. Acids. Res. 30 (2002) e122

13. Rose, J.A., Deaton, R.J., Suyama, A.: Statistical thermodynamic analysis and design of oligonucleotide based computers. Natural Computing 3 (2004)

14. SantaLucia, Jr., J., Hicks, D.: The thermodynamics of DNA structural motifs. Annu. Rev. Biophy. Biomolec. Struct. 33 (2004) 415-40

15. Kubota, M., Ohtake, K., Komiya, K., Sakamoto, K., Hagiya, M.: Branching DNA machines based on transitions of hairpin structures. In: Proc. Congr. Evol. Comp. (CEC'03). (2003) 2542-2548 